\section{Kardioversion: Edoxaban als Thromboseschutz wirksam und sicher}

Die Kardioversion von Vorhofflimmern erfordert immer eine effektive Antikoagulation, da ein erhöhtes Risiko für eine thromboembolische Komplikation besteht. Einer neuen Studie zufolge ist der Faktor-Xa-Hemmer Edoxaban eine zumindest gleichwertige Alternative zum herkömmlichen Vorgehen mit niedermolekularem Heparin und Vitamin-K-Antagonist.

Eür die Kardioversion des VorhofflimF merns gibt es nach der offiziellen Leitlinie zwei Strategien: die konventionelle nach einer dreiwöchigen Antikoagulation oder die frühe nach Ausschluss eines Thrombus mittels TEE. Beides ist gleich wirksam und gleich sicher bei effektiver Antikoagulation. Dauert das Vorhofflimmern nicht länger als 48 Stunden an, kann die elektrische oder medikamentöse Kardioversion auch sofort durchgeführt werden, wobei gleichzeitig die Antikoagulation eingeleitet wird.

„Eine Kardioversion ist immer mit einem Risiko für ein thromboembolisches Ereignis, sprich Schlaganfall, assoziiert, sodass eine effektive Antikoagulation zwingend erforderlich ist", betonte Prof. Andreas Götte, Chefarzt der kardiologischen Abteilung im St. Vincenz Krankenhaus in Paderborn. Nach der erfolgreichen Kardioversion müsse die Antikoagulation immer über mindestens diziert.

\section{ENSURE-AF-Studie: Kein \\ Unterschied}

drei Wochen fortgeführt werden, da es einige Wochen dauert, bis sich der Vorhof auch mechanisch erholt hat. Doch bei Patienten mit einem CHA2DS2-VASc-Score von mindestens 2 Punkten bei Männern und 3 Punkten bei Frauen ist eine dauerhafte Antikoagulation unabhängig vom Erfolg der Kardioversion in-

Das bisherige Vorgehen bei der Kardioversion besteht in der Gabe eines niedermolekularen Heparins überlappend mit einem Vitamin-K-Antagonisten, bis der INR-Zielwert $>2$ erreicht ist. Der Frage, ob eine Antikoagulation mit einem NOAK, genauer gesagt dem Faktor-XaHemmer Edoxaban (Lixiana ${ }^{\star}$ ), auch in dieser Situation wirksam und sicher ist, wurde im Rahmen der ENSURE-AF (EdoxabaN vs. warfarin in subjectS UndeRgoing cardiovErsion of Atrial

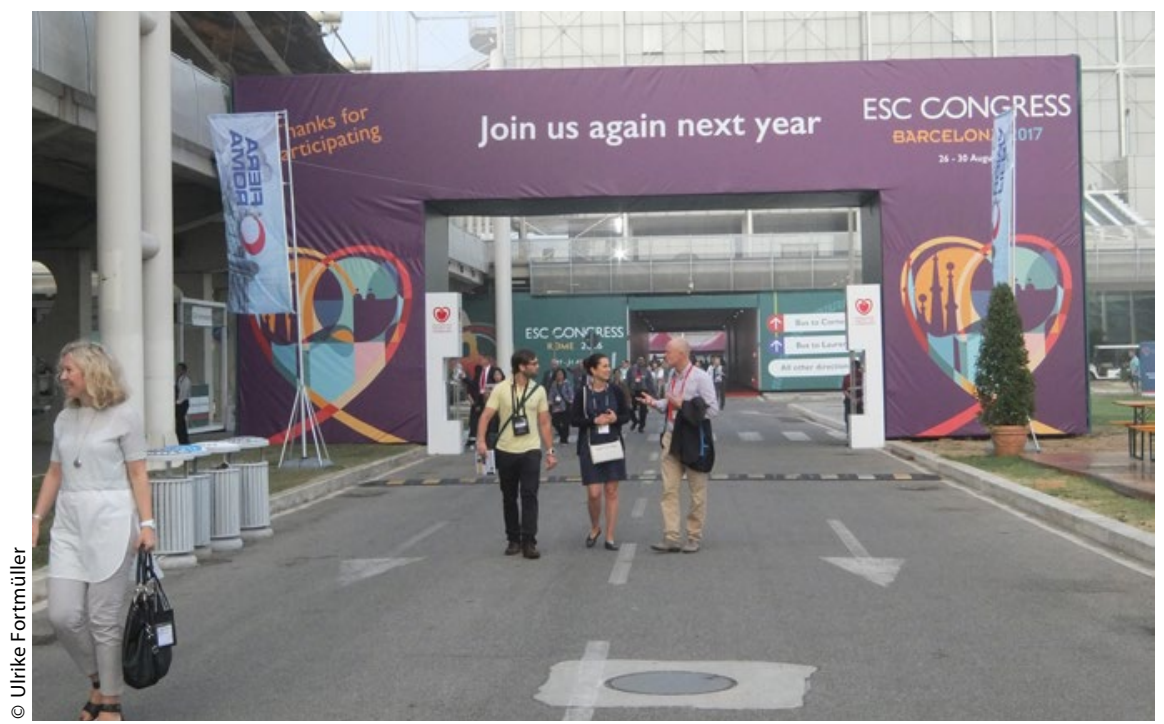

Im nächsten Jahr wird der ESC-Kongress in Barcelona stattfinden - hoffentlich mit weniger Wartezeiten.
Fibrillation)-Studie nachgegangen. Die Ergebnisse wurden beim ESC-Kongress erstmals vorgetragen und gleichzeitig im „The Lancet“ publiziert. ENSUREAF ist mit 2.199 Patienten die größte jemals durchgeführte Kardioversionsstudie.

Die Patienten erhielten randomisiert entweder 60 mg Edoxaban einmal täglich oder Enoxaparin überlappend mit Warfarin. Bei Patienten mit einer GFR $<50 \mathrm{ml} / \mathrm{min}$ oder einem Köpergewicht $\leq 60 \mathrm{~kg}$ oder einer P-GP-Inhibitor-Therapie wurde die Enoxaparin-Dosis auf $30 \mathrm{mg}$ reduziert.

„Insgesamt ergab sich kein signifikanter Unterschied weder im Hinblick auf die Wirksamkeit noch die Sicherheit zwischen den beiden Patientengruppen", berichtete Götte. Die Rate für den primären Endpunkt (Schlaganfall, systemische Embolie, Myokardinfarkt und kardiovaskulärer Tod) lag unter Edoxaban bei $0,5 \%$, unter $\mathrm{NMH} /$ Warfarin bei 1,0\%. Dieses Ergebnis war unabhängig davon, ob die TEE-gesteuerte Strategie mit früher Kardioversion oder die konventionelle Vorgehensweise mit der Kardioversion nach dreiwöchiger Antikoagulation gewählt wurde.

Die Blutungsrate war insgesamt sehr gering und auch hier ergaben sich keine signifikanten Unterschiede zwischen den beiden Strategien. Die Rate an stärkeren Blutungen lag unter Edoxaban bei $1,5 \%$, bei NMH/Warfarin bei $1,0 \%$, die Gesamtblutungsrate bei 3,0 vs. $3,2 \%$.

Insgesamt ergab sich ein Nettobenefit für die Edoxaban-Gruppe, und dieser war auch unabhängig von der Nierenfunktion und der gewählten Dosis.

„Die Ergebnisse sprechen eindeutig dafür, dass Edoxaban auch bei der Kardioversion eine wirksame und sichere Option darstellt, die der bisherigen Strategie keinesfalls unterlegen ist“, schloss Götte. Dr.med. Peter Stiefelhagen

Hot Line "New preventive Strategies" am 30. August 2016, ESC-Kongress in Rom

Götte $A$ et al. Edoxaban versus enoxaparin-warfarin in patients undergoing cardioversion of atrial fibrillation (ENSURE-AF): a randomised, open-label, phase $3 \mathrm{~b}$ trial. Lancet 2016, online 30 August; DOI: 10.1016/S0140-6736(16)31474-X 